\title{
Computationally efficient optic nerve head detection in retinal fundus images
}

\author{
Reza Pourreza-Shahri ${ }^{2}$, Meysam Tavakoli ${ }^{1}$, Nasser Kehtarnavaz ${ }^{2}$ \\ Oklahoma State University, University of Texas at Dallas
}

\begin{abstract}
To appear in Biomedical Signal Processing and Control, Volume 11, May 2014, Pages 63-73
https://www.sciencedirect.com/science/article/abs/pii/S1746809414000378
\end{abstract}

\begin{abstract}
This paper presents a computationally efficient method for the detection of optic nerve head in both color fundus and fluorescein angiography images. It involves a combination of Radon transformation and multi-overlapping windows within an optimization framework in order to achieve a robust detection in the presence of various structural, color, and intensity variations in such images. Three databases have been examined and it is shown that the introduced method provides high detection rates while achieving faster proceeding rates than the existing algorithms that possess comparable detection rates.
\end{abstract}

Index Terms-Optic nerve head detection, Radon transformation, fundus image, fluorescein angiography, diabetic retinopathy.

\section{INTRODUCTION}

Computer Assisted Diagnosis (CAD) of retinopathy is currently being used to lower the workload of ophthalmologists as it is providing a non-labor intensive approach to the detection of the anatomical landmarks and lesions in retinal images. The computationally efficiency of such a CAD system would allow the screening of more patients within existing clinic or hospital time constraints.

The localization of retinal landmarks in particular Optic Nerve Head (ONH) constitutes the initial step towards identifying pathological conditions. Detection of $\mathrm{ONH}$ plays a key role in Diabetic Retinopathy (DR) [1, 2]. ONH is a yellowish region in a color fundus image occupying one seventh of the fundus image [3] (see Fig. 1). The main characteristic of $\mathrm{ONH}$ is its rapid intensity variation due to dark blood vessels that are in the vicinity of $\mathrm{ONH}$. ONH has three characteristics that have been used for its localization: (1) it appears as a bright disk nearly $1600 \mu \mathrm{m}$ in diameter; (2) large blood vessels enter it from above and below; and (3) blood vessels diverge from it.

In many approaches, the ONH diameter is used as a length reference to measure objects in retina [1, 4]. In [3], the relatively constant distance between $\mathrm{ONH}$ and fovea was used to estimate the location of the latter. In [5], masking of $\mathrm{ONH}$ improved the classification of exudates regions and decreased the false positive rate. However, as noted in [6], detection of $\mathrm{ONH}$ is challenging due to the discontinuity of its boundary caused by large vessels as well as its considerable color or intensity variations as a result of structures such as exudates. The change in the color, shape, and depth of $\mathrm{ONH}$ provides a pathological sign, in particular a sign of glaucoma [7]. Glaucoma is the second most common cause of blindness in the world [8].

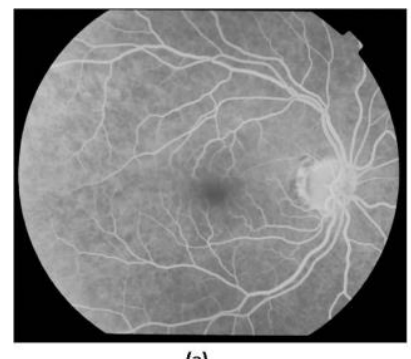

(a)

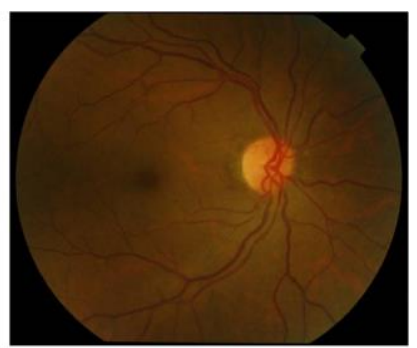

(b)
Fig. 1. Fundus image from same eye: (a) fluorescein angiography and (b) color fundus image.

\section{PREVIOUS WORKS}

There are several existing algorithms that determine the location (generally center) of $\mathrm{ONH}$ or its boundary. Sinthanayothin et al. [3] used the area with the highest average intensity variation to detect $\mathrm{ONH}$ using an adaptive local contrast enhancement method. Walter and Klein [9] obtained the ONH center as the center of the brightest connected object in a fundus image instead of using intensity variation via the watershed transformation of the gradient image. Foracchia et al. [10] used the convergence of vessels to detect the $\mathrm{ONH}$ center. Youssif et al. [11] utilized the directional pattern of the retinal blood vessels for the detection of ONH. Their method involved normalizing luminosity and contrast throughout the image using illumination equalization and adaptive histogram equalization methods. Lu and Lim [12] located $\mathrm{ONH}$ based on its bright appearance in a color fundus image using a set of concentric lines with different directions and evaluated the image variation along multiple directions. The detection of $\mathrm{ONH}$ was achieved via the orientation of the line segment with the maximum or minimum variation.

A number of segmentation-based methods have also appeared in the literature. Li and Chutatape [13, 14] used an active shape model to detect $\mathrm{ONH}$ [15]. An active contour model was also discussed in $[4,16]$ by Osareh et al. [4, 16] to detect ONH. Lowell et al. [17] designed an ONH template and correlated it to the intensity component of the fundus image using the full Pearson-R correlation. Another model-based approach was presented by $\mathrm{Xu}$ et al. 
in [18], where clustering-based classification of contour points was integrated into an active contour formulation. Wong et al. [19] used the level-set technique followed by ellipse matching. They obtained the $\mathrm{ONH}$ location by means of histogram analysis and a modified version of the conventional level-set method using the red channel.

Furthermore, $\mathrm{Lu}$ [20] designed a circular transformation to capture simultaneously both the circular shape of $\mathrm{ONH}$ and the image variation across the $\mathrm{ONH}$ boundary. A Hausdorff-based template matching together with a pyramidal decomposition were proposed by Lalonde et al. in [21]. Frank ter Haar [22] used illumination equalization in the green channel to address the difficulty of pyramidal decomposition in dealing with large areas of bright pixels.

Some ONH localization techniques not only use the $\mathrm{ONH}$ characteristics, but also exploit the location and orientation of vessels [23-27]. For example, Niemeijer et al. [27] presented the use of local vessel geometry and image intensity features. Tobin et al. [28] applied a method that mainly relied on vessels related to the $\mathrm{ONH}$ characteristics. A Bayesian classifier was used to classify each pixel in red-free images. Abramoff and Niemeijer [29] utilized the same $\mathrm{ONH}$ characteristics via $\mathrm{kNN}$ regression for the detection of $\mathrm{ONH}$. The method introduced by Abramoff $e t$ al. [30] involved a pixel classification approach. In a recent study, Hsiao et al. localized $\mathrm{ONH}$ by an illumination correction operation, and contour segmentation via a supervised gradient vector flow snake [31]. Yu et al identified ONH candidates by first using template matching and then by using the vessel characteristics [32]. Finally, some techniques have made use of the fact that major retinal vessels converge into $\mathrm{ONH}[10,11,22,33]$. Many existing methods have reported noticeable failure for fundus images with a large number of white lesions, light artifacts or strongly visible choroidal vessels [34].

In this paper, a new ONH detection method based on Radon Transform (RT) and multi-overlapping windows is introduced. The key attribute of this new method is its computational efficiency. Two publicly available databases are examined: the DRIVE database [35], and the STARE dataset [36]. Noting that some retinal lesions, such as exudates, have intensity and color that are similar to $\mathrm{ONH}$ and thus can affect the performance of an automated $\mathrm{ONH}$ detection system, we have also considered DR images in color fundus images in the database MUMS-DB (Mashhad University of Medical Sciences image Database). In addition, fluorescein angiography (FA) fundus images in MUMS-DB are examined.

In the next section, the details of our algorithm for the detection of $\mathrm{ONH}$ are described.

\section{INTRODUCED METHOD}

An RT-based algorithm is proposed here to detect $\mathrm{ONH}$ in fundus images. RT provides lower noise or intensity variation sensitivity due to the integration involved in it. A fundus image is first partitioned into overlapping blocks or sub-images. Local RT is then applied to each block or subimage. The sub-images exhibiting peaks in the Radon space are then further processed in order to locate $\mathrm{ONH}$. The pipeline of the method is illustrated in Fig. 2.

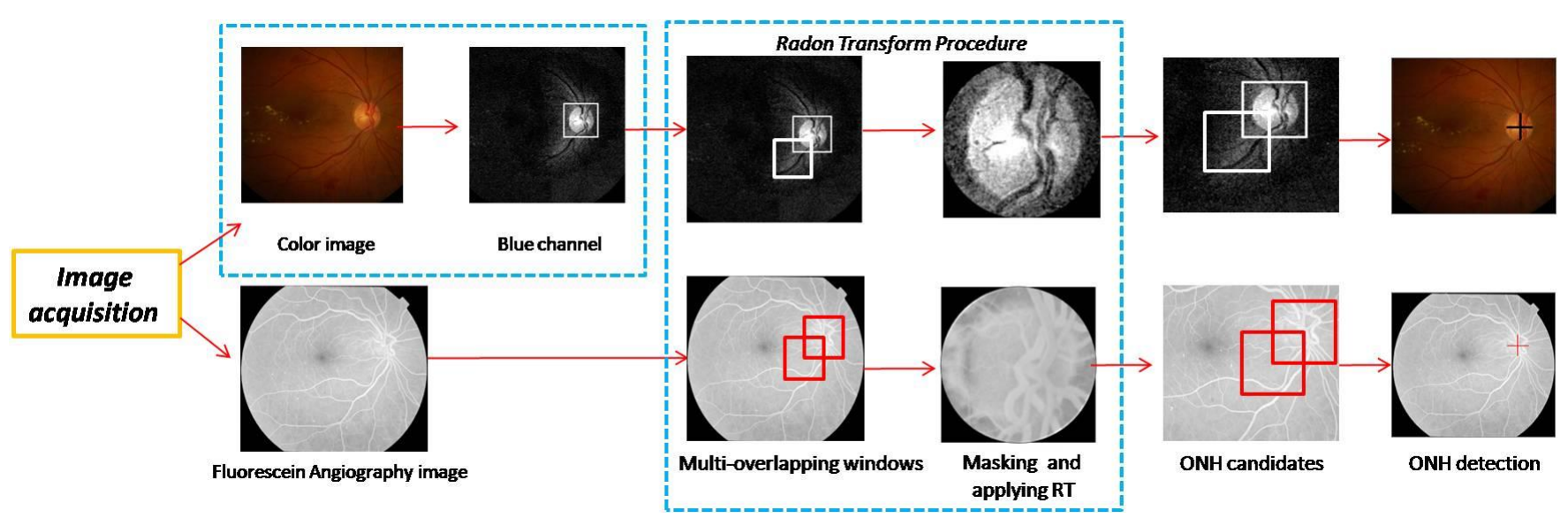

Fig. 2. Pipeline of the introduced method

The main components of the detection algorithm, the $\mathrm{ONH}$ detection procedure, and its computational efficiency aspect are covered in the following subsections A, B and C, respectively.

\section{A. Algorithm components}

The algorithm contains three main components as follows.

\section{Fundus region detection}

At first, it is important to separate fundus from background (or region that is out of the fundus field). A fundus image consists of a circular fundus and a dark background surrounding the fundus. Fundus is considered the region- 
of-interest and the processing is not applied to the background region. There are fundus masks that are supplied with retinal images in the databases examined. In a fundus mask, fundus pixels are marked with 1's and the background with 0's. With the help of the fundus mask, the detection algorithm would only process the pixels belonging to the fundus and not the background (see Fig. 3).
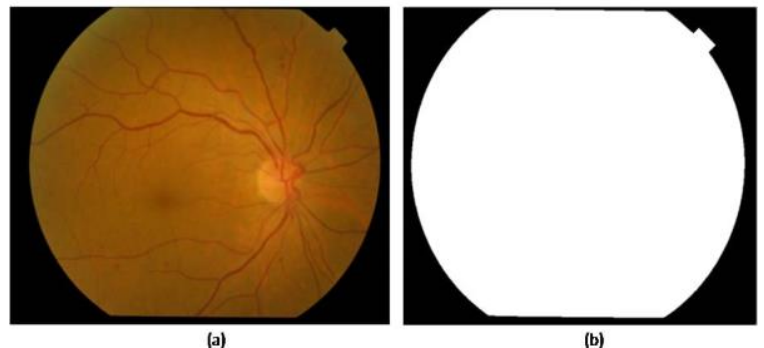

Fig. 3. (a) Fundus image, and (b) fundus mask

\section{Multi-overlapping window}

In our introduced method, a fundus image is partitioned into widows or sub-images. To find objects on the border of sub-images, overlapping sliding windows are considered. The size of the targeted object or ONH $(n)$ is used to determine the size of the sub-image or sliding window. A parameter that has influence on the outcome is the windows overlapping ratio (step). If this ratio is equal to one, every pixel is examined just one time and subimages would have no overlap. If the step is defined as two or more, then each pixel is examined ( $n / s t e p)$ times either in horizontal or vertical sliding direction and this way each pixel is considered in up to $n^{2}$ sub-images (see Fig. 4).

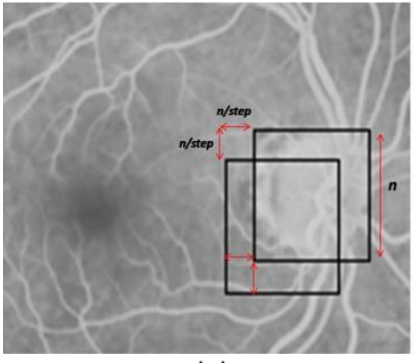

(a)

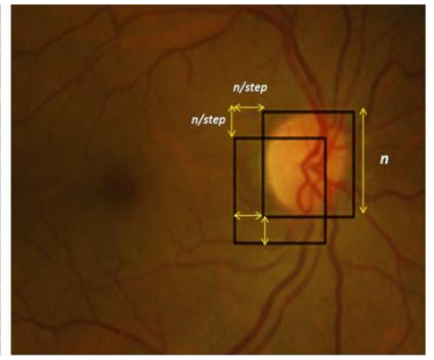

(b)
Fig. 4. Window size and overlapping ratio (n, step) in (a) fluorescein angiography, and (b) color fundus image

\section{Radon Transform}

Radon Transform (RT) is widely used in X-ray computed tomography (CT). A projection of a 2D function $f(x, y)$ corresponds to a set of line integrals. The Radon function computes the line integrals from multiple parallel paths or beams. The beams are spaced 1 pixel unit apart. To represent an image, the Radon function takes multiple, parallel-beam projections of the image from different angles by rotating the object of interest around the center of the image. Fig. 5 shows a single projection at a specified rotation angle.

Let us consider the RT of a planar function $f$ :

$$
\begin{aligned}
\check{f}_{\theta}(s)=\int_{-\infty}^{+\infty} f(x, y) d s \\
=\int_{-\infty}^{+\infty} f(s(\cos \theta+\sin \theta) \\
+z(-\sin \theta+\cos \theta)) d s
\end{aligned}
$$

where

$$
\left[\begin{array}{l}
x \\
y
\end{array}\right]=\left[\begin{array}{cc}
\cos \theta & \sin \theta \\
-\sin \theta & \cos \theta
\end{array}\right]\left[\begin{array}{l}
S \\
Z
\end{array}\right]
$$

Then, the RT of a continuous two-dimensional function $f$ becomes

$$
\begin{gathered}
\check{f}_{\theta}(s)=\int_{-\infty}^{+\infty} \int_{-\infty}^{+\infty} f(x, y) \delta(s-x \cos \theta \\
-y \sin \theta) d x d y
\end{gathered}
$$

A single projection of the object $\check{f}_{\theta}(s)$ is stated by Equation (2), where the Dirac delta function $\delta$ defines the path of the line integral. Equation (2) expresses the relationship between the object function $f(x, y)$ and the measured projection $\check{f}_{\theta}(s)$. The projection $\check{f}_{\theta}(s)$ in Equation (2) may be interpreted as the one-dimensional function $\check{f}_{\theta}(s)$ of a single variable $s$ with $\theta$ as a parameter. With the arrangement exhibited in Fig. 5, $\check{f}_{\theta}(s)$ is referred to as a parallel projection.

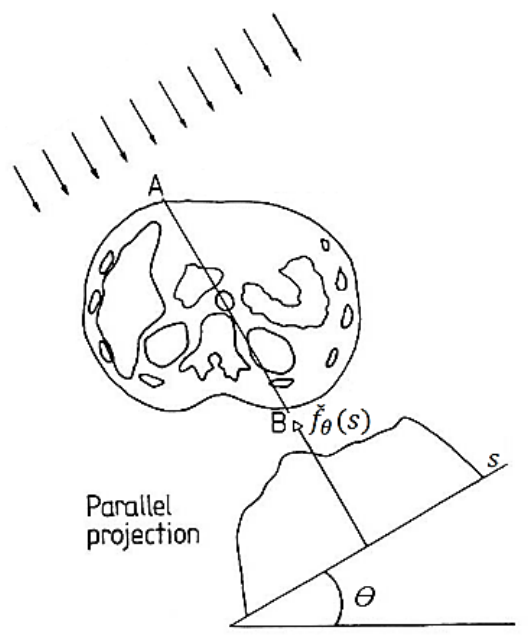

Fig. 5. Parallel projection line integral

$\mathrm{RT}$ is able to transform a pattern to a line in the Radon space and thus allows it to be easily distinguished from other patterns.

\section{B. ONH detection}


$\mathrm{ONH}$ is characterized by its bright circular characteristic and because of large vessels coming out and going into it, its edges are ill defined. $\mathrm{ONH}$ appears non-uniform in intensity, size, and location.

Our introduced algorithm for $\mathrm{ONH}$ detection in both FA and color fundus images comprises 4 steps:
1. Image partitioning
2. Applying Radon Transform
3. ONH validation
4. ONH detection

\section{Step 1) Image partitioning}

$\mathrm{ONH}$ is detected in local windows. The window size $(n)$ has an effect on the detection outcome. Let

$$
I=\left\{w\left(x_{i}, y_{j}\right) \mid i=1 \ldots N, j=1 \ldots M\right\}
$$

denote the sub-images $w\left(x_{i}, y_{j}\right)$ of size $n \times n$. If $n$ is selected too small or too large, it would lead to extracting patterns other than ONH such as Microaneurysms or scar tissue. Based on our prior knowledge of the ONH size and its maximum diameter, an appropriate $n$ is selected here. The size of $n$ is chosen equal to the maximum diameter of $\mathrm{ONH}$ in a database. If the resolution of images is different, $n$ is selected accordingly. For example, for the MUMS-DB database having an image resolution of $2896 \times 1944, n$ is chosen to be 313 pixels, while for the DRIVE database, $n$ is chosen to be 79 pixels and for the STARE database, $n$ is chosen to be 130 pixels.

The window size is also determined automatically based on the scale factor of images under analysis. Given the actual size of $\mathrm{ONH}$ in $\mathrm{mm}$, one can estimate the $\mathrm{ONH}$ size in pixels and subsequently the window size.

Another important parameter is the windows overlapping ratio (step). The processing speed depends on this step parameter. As step is increased, the computation time increases exponentially. In the results reported in this paper, step was chosen to be 4 meaning $1 / 4$ size window overlap.

\section{Step 2) Applying Radon Transform}

High intensity differences between $\mathrm{ONH}$ and background in FA and color images cause $\mathrm{ONH}$ to be associated with peaks in the Radon space. Moreover, in color images, the blue (B) component of sub-images is selected for applying RT to detect ONH due to its high contrast between its ONH and background. In the $\mathrm{B}$ channel, the red features like vessels, Microaneurysms, and Hemorrhages have low contrast and only yellowish pattern of $\mathrm{ONH}$ or exudates have high contrast. In other words, the $\mathrm{B}$ component provides a good separation for the white and yellow colors in comparison to the background (see Fig. 6).

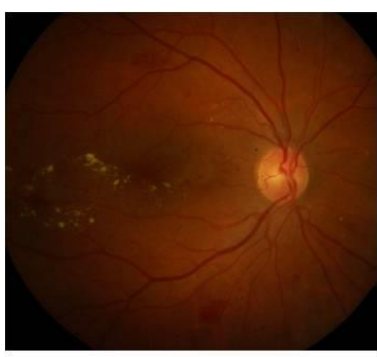

(a)

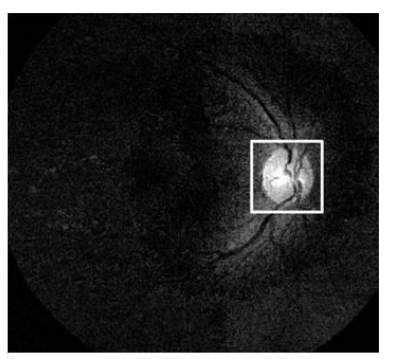

(b)
Fig. 6. (a) Original fundus image, (b) its blue channel and sub-image covering the $\mathrm{ONH}$

After generating the sub-images into windows or blocks, the RT is applied to the masked image as noted in Equation (2).

The amplitude of the projection along the diagonal directions $\left(\theta=45^{\circ}, \theta=135^{\circ}, s=n \sqrt{2}\right)$ is higher than other directions; thus, the peak of RT occurs mostly along diagonal directions. To eliminate the diagonal effect, the sub-images are first masked via a circular mask. The masking process is illustrated in Figs. 7 and 8.

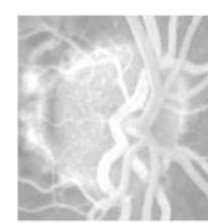

(a)

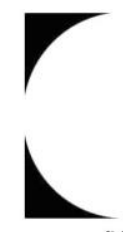

(b)

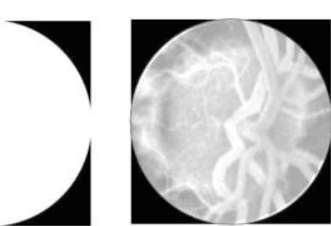

(c)
Fig. 7. Masking process in FA: (a) original sub-image, (b) applied mask, (c) masked sub-image

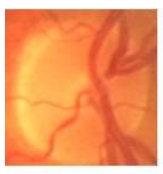

(a)

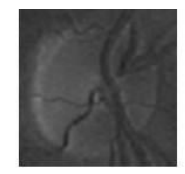

(b)

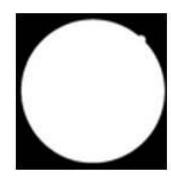

(c)

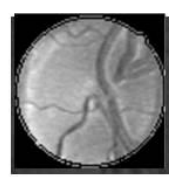

(d)
Fig. 8. Masking process in color: (a) original sub-image, (b) blue component, (c) applied mask, (d) masked sub-image

As mentioned earlier, $\mathrm{ONH}$ in a sub-image is associated with a prominent peak in the Radon space. Hence, at this stage, it is required to detect peaks (see Fig. 8). Peaks are detected in the Radon space and the profiles in which they occur serve as candidates for $\mathrm{ONH}$. These profiles are further analyzed for validation of candidate ONHs. All subimages which have a peak profile higher than a predefined threshold are compared. In the results reported in this paper, the threshold was considered to be 0.9 of the largest Radon projection in the sub-image. In other words, initially all Radon projections for one sub-image are found and then the maximum is found. If the peak of RT along all the projection angles is greater than 0.9 of the largest RT projections in the sub-image, that sub-image is regarded as a candidate that may contain the $\mathrm{ONH}$. 

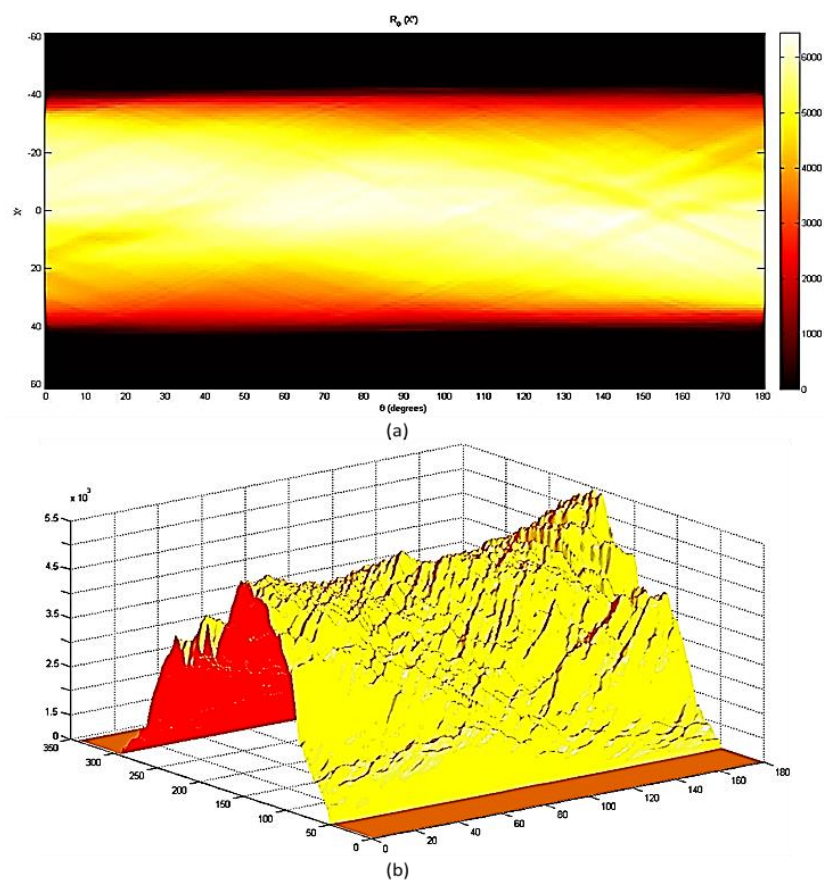

Fig. 9. (a) 2D, and (b) 3D image resulting from Radon transformation of a masked sub-image containing $\mathrm{ONH}$

\section{Step 3) ONH validation}

The concept of validation is similar to peak measuring along all the projection angles in Radon transformation of a sub-image with a central point pattern (see Fig. 9). Based on this approach, all the sub-images which have a peak profile higher than a predefined threshold get evaluated.

To detect $\mathrm{ONH}$, the RT property for round objects is used. For a round object, RT provides the same profile along all the directions. Due to the roundness of $\mathrm{ONH}$, the profiles related to the projections do not differ much. As a result, one can detect the sub-image that contains the ONH. This is achieved by computing the mean square error (MSE) between the projections. The MSE between the projections inside the blocks is used as a similarity measure for the Radon peaks. In other words, the sub-image which minimizes the MSE among all of its different projections is found (see Fig. 10 and Fig. 11).

The MSE is computed as follows:

$$
\operatorname{MSE}(r)=\frac{1}{m \cdot n} \sum_{i=1}^{n} \sum_{j=1}^{m}(R(i, j)-R(r, j))^{2}
$$

where $R(i, j)$ denotes the $(i, j)^{\text {th }}$ component of the Radon matrix $(R), M$ and $N$ are the dimensions of $R$ and $r$ is the index of the reference column in $R$.

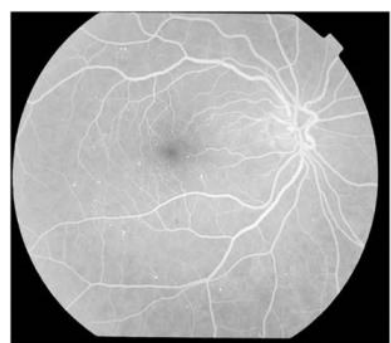

(a)

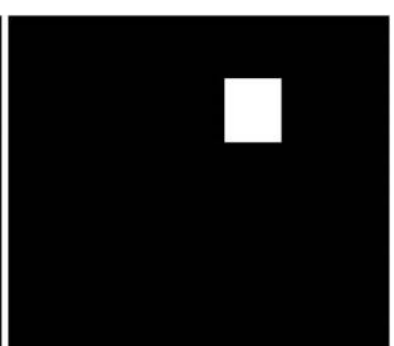

(b)
Fig. 10. (a) Original fluorescein angiography fundus image from MUMS$\mathrm{DB},(\mathrm{b})$ its validated $\mathrm{ONH}$ sub-image

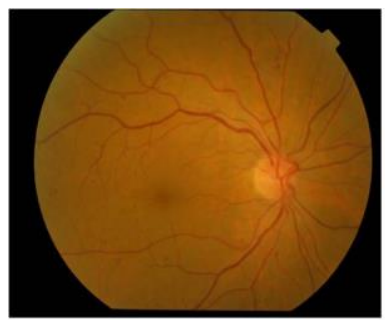

(a)

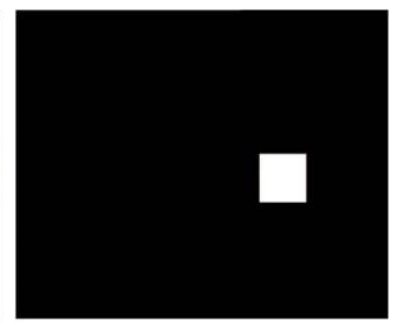

(b)
Fig. 11. (a) Original fundus image from MUMS-DB, (b) its validated ONH sub-image

\section{Step 4) ONH detection}

$\mathrm{ONH}$ detection and its changes are quite important in identifying some pathologic conditions. Contrast variance of $\mathrm{ONH}$ could generate some artifacts in image segmentation of fundus images. Thus, it is necessary to find $\mathrm{ONH}$ and mask it. After the above validation of $\mathrm{ONH}$, the center of the sub-image which touches the $\mathrm{ONH}$ is considered to be the center of the ONH. Some sample results in FA and color fundus images from the three databases examined are shown in Figs. 12 through 15.

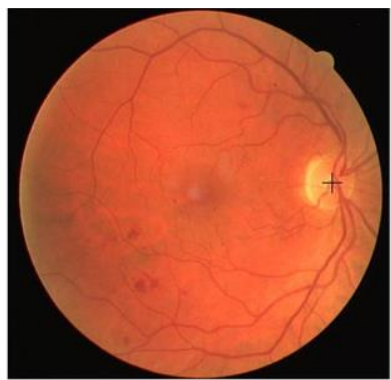

(a)

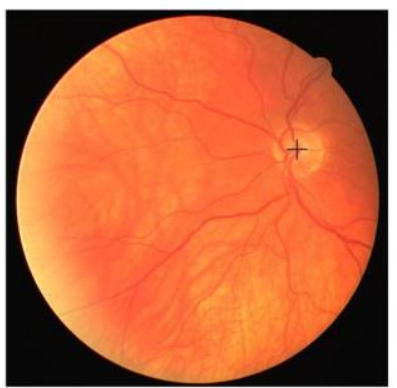

(c)

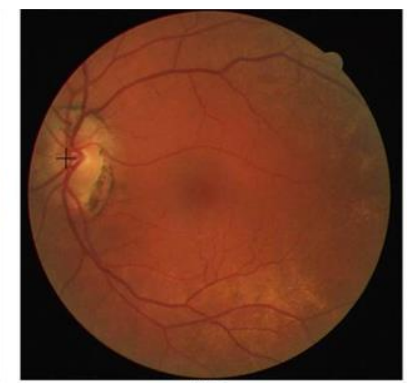

(b)

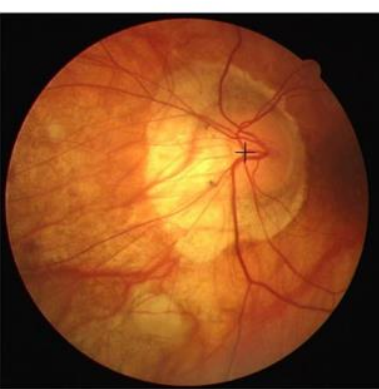

(d) 
Fig. 12. Outcome of the introduced method applied to sample images of DRIVE database image (black cross represents the estimated ONH center).

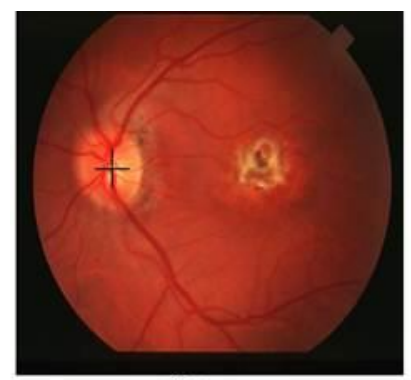

(a)

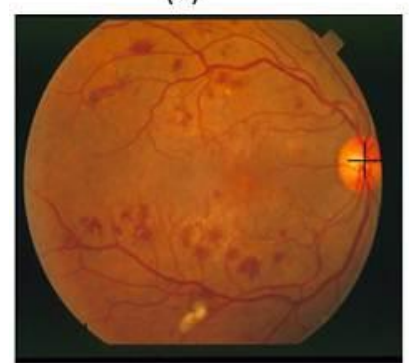

(c)

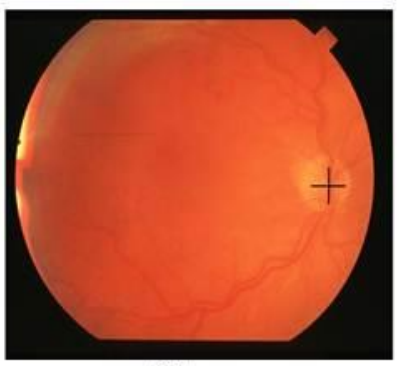

(e)

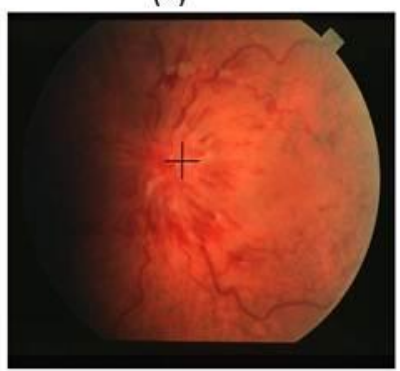

(g)

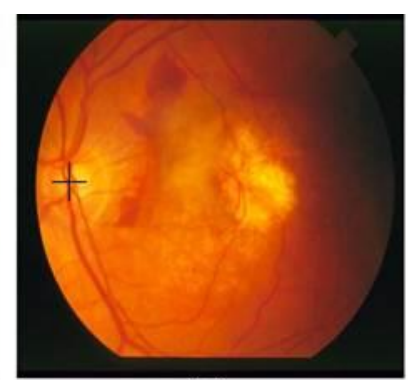

(b)

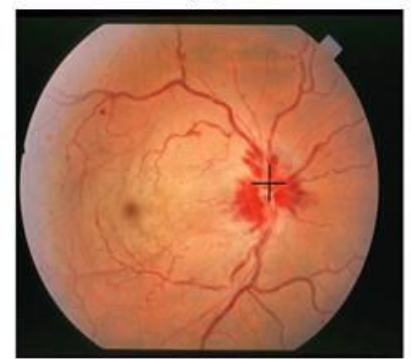

(d)

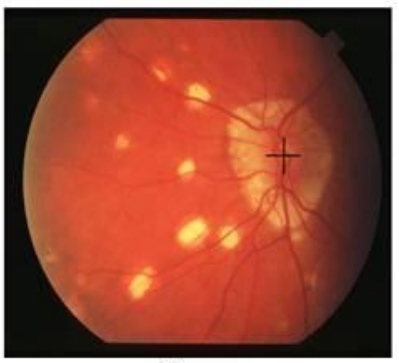

(f)

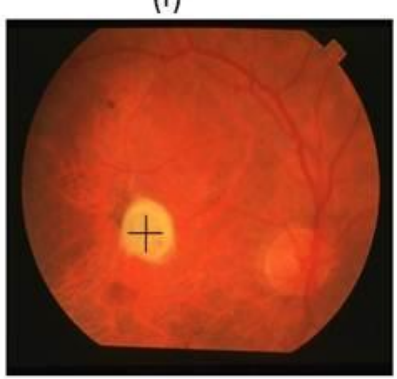

(h)
Fig. 13. Outcome of the introduced method applied to sample images of STARE database (black cross represents the estimated ONH center): (a) (g) correct detection, (b) h failure case of the detection

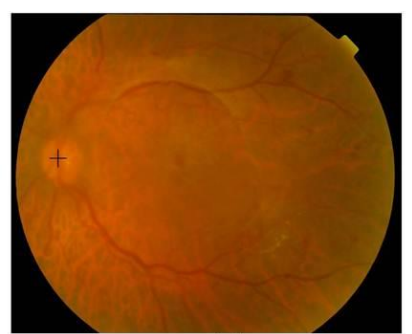

(a)

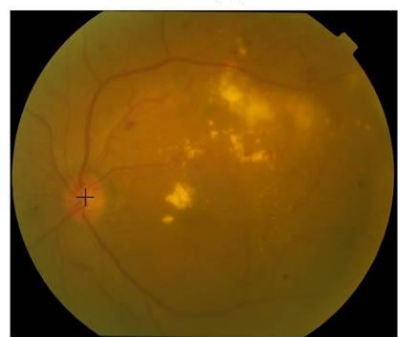

(c)

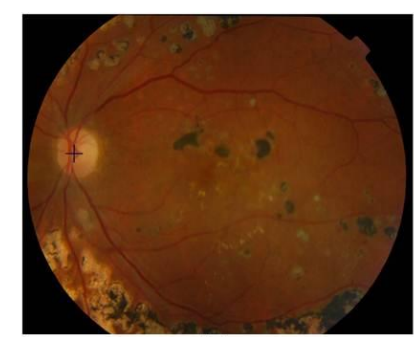

(b)

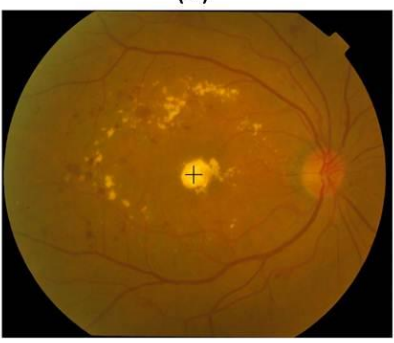

(d)
Fig. 14. Outcome of the introduced method applied to sample images of MUMS-DB color database (black cross represents the estimated ONH center): (a)-(c) correct detection, (b) d failure case of the detection

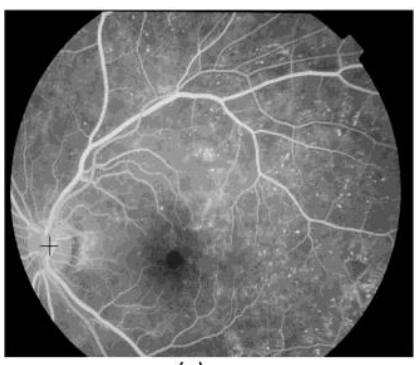

(a)

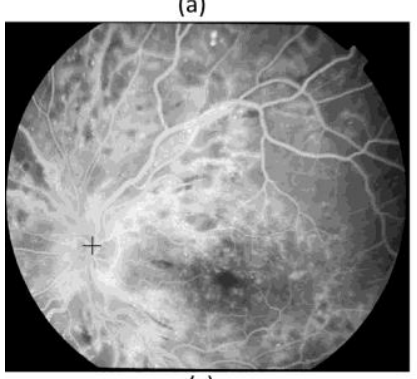

(c)

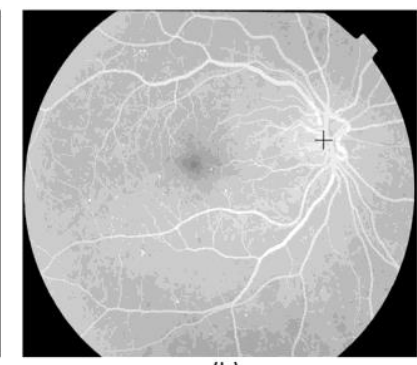

(b)

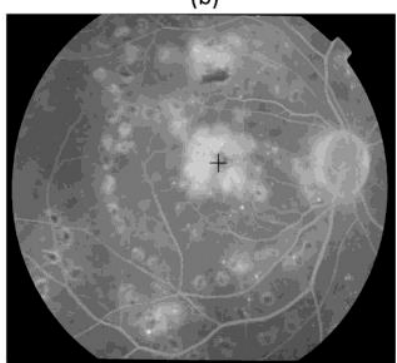

(d)
Fig. 15. Outcome of the introduced method applied to sample images of MUMS-DB FA database (black cross represents the estimated ONH center): (a)-(c) correct detection, (b) d failure case of the detection

\section{Computational complexity}

The main attribute of our algorithm is its computational efficiency compared with other algorithms. If the input image is size of $M \times N$, the window of size $n$ with the step $s$, the number of operations is as follows:

Fundus area detection: $N M$

Radon Transform: $9 M N s^{2} a$, where $a$ indicates the number of projections in Radon Transform

Validation: 3anc, where $c$ indicates the number of candidates 
Hence, the total number of operations equals $N M\left(1+9 a s^{2}\right)$ $+3 a n c$.

\section{RESULTS AND DISCUSSION}

Our algorithm was written in MATLAB and applied to the color retinal images in the public DRIVE and STARE databases and to the local database MUMS-DB which include normal and diabetic FA and color retinal images. The DRIVE database consists of 40 images in which 33 images do not have any sign of DR and 7 images show signs of early or mild DR. The image resolution is 564 by 584 pixels acquired using a Canon CR5 3CCD camera with a $45^{\circ}$ field of view (FOV). The STARE database consists of 81 fundus images that were used initially by Hoover and Goldbaum [36] to assess their automatic ONH localization technique. The images were captured using a TOPCON TRV 50 retina camera at $35^{\circ}$ FOV with an image resolution of $700 \times 605$ pixels. This dataset contains 31 images of normal retina and 50 images of diseased retina. The MUMS-DB database consists of more than 2000 retinal images (FA and color). All images were obtained using a TOPCON (TRC-50EX) retinal camera at $50^{\circ}$ FOV. Images were mostly obtained from the posterior pole view including optic disc and macula. Their image resolution is $2896 \times 1944$ pixels.

We examined 120 images for each image type (120 for FA and 120 for color) including 100 cases in different stages (4 stages) of DR, and 20 without DR with no systemic disease or ocular micro-vascular involvement. The selection and diagnosis was done by an ophthalmologist in a blind fashion. The ophthalmologist was asked to mark the ONHs in these images after adding the transparent layer to the images. The $\mathrm{ONH}$ locations were saved in similar size files to form the ground truth files. The ground truth files of selected fundus images were also collected. Based on the manual or visual ONH detection, our algorithm found the true locations of $\mathrm{ONH}$ in 117 out of 120 color images $(97.5 \%)$ and 110 out of 120 in FA images $(91.3 \%)$ of the
MUMS-DB database. Some examples of the ONH detection for both the FA and color images of the MUMSDB database are shown in Figs. 14 and 15.

Additionally, the $\mathrm{ONH}$ location was detected correctly in all of the 40 images of the DRIVE database (100\%) using our method (see Fig. 12). Our method detected the ONH locations correctly in 78 out of the 81 images of the STARE dataset (96.3\%), (see Fig. 13).

TABLE I

DATABASESAND ACCURACIES

\begin{tabular}{|c|c|c|c|}
\hline Database & Images & $n$ & Accuracy \\
\hline $\begin{array}{c}\text { MUMS-DB } \\
\text { (color) }\end{array}$ & 120 & 313 & $97.5 \%$ \\
\hline $\begin{array}{c}\text { MUMS-DB } \\
\text { (FA) }\end{array}$ & 120 & 313 & $91.3 \%$ \\
\hline DRIVE & 40 & 79 & $100 \%$ \\
\hline STARE & 81 & 130 & $96.3 \%$ \\
\hline
\end{tabular}

The estimated ONH center was considered to be correct if it was positioned within 60 pixels of the manually identified center, as proposed in [10]. The average distance (for the 69 successful images) between the estimated ONH center and the manually identified center was 10 pixels and for the 9 successful images, this distance was 21 pixels in the STARE dataset. In the DRIVE database, the average distance of 40 successful images between the estimated $\mathrm{ONH}$ center and the manual segmentation was 13 pixels. Finally, the average distance of the successful images in the MUMS-DB database was 14 pixels. The cases in which the ONHs were not correctly detected were due to uneven round-shaped illumination that biased the ONH candidates (see Figs. 13(h), 14(d), and 15(d)).

Table II compares the accuracies of the introduced method and two representative existing methods which have been reported to have the highest detection rates and at the same the fastest detection times.

TABLE II

COMPUTATIONAL COMPLEXITY COMPARISON OF ONH DETECTION METHODS AND DETECTION RATES FOR STARE DATABASE

\begin{tabular}{|c|c|c|c|c|c|}
\hline Methods & Detection Rate & $\begin{array}{l}\text { No. of failed } \\
\text { images }\end{array}$ & $\begin{array}{l}\text { Accuracy } \\
\text { (pixels) }\end{array}$ & $\begin{array}{l}\text { Computational complexity in terms } \\
\text { of approximate number of operations }\end{array}$ & Notations \\
\hline $\begin{array}{l}\text { Foracchia et } \\
\text { al.[10] }\end{array}$ & $97.5 \%$ & 2 & 23 & $24 M N W^{2}+180 I V$ & $\begin{array}{c}W=16 \\
V=300 \\
I: \text { no of iterations in } \\
\text { simulated annealing }\end{array}$ \\
\hline $\begin{array}{l}\text { Youssif et } \\
\text { al. [11] }\end{array}$ & $98.8 \%$ & 1 & 26 & $\begin{array}{c}M N\left(W_{1}^{2}+2 W_{2}^{2}+24 W_{3}^{2}+15\right)+ \\
324 V W_{4}^{2}\end{array}$ & $\begin{aligned} & W_{1}=40 \\
& W_{2}=80 \\
& W_{3}=15 \\
& W_{4}=40 \\
& V: \text { Number of vessel pixels }\end{aligned}$ \\
\hline $\begin{array}{c}\text { Mahfouz } \\
{[33]}\end{array}$ & $92.6 \%$ & $? ?$ & 14 & $M N(10+V)+2 M W^{2}+4 W^{2}$ & $\begin{array}{l}W: \text { ONH size (130) } \\
V: \text { Vessel width }\end{array}$ \\
\hline
\end{tabular}




\begin{tabular}{|c|c|c|c|c|c|}
\hline $\begin{array}{c}\text { Introduced } \\
\text { method }\end{array}$ & $96.3 \%$ & 3 & 10 & $N M\left(1+9 a s^{2}\right)+3 a n c \quad s=4$ & \\
& & & \\
$n=130$ & 12 \\
\hline
\end{tabular}

$M$ and $N$ are 605 and 700 , respectively, in the STARE dataset. As can be seen from Table II, the number of operations in our algorithm is much less than Foracchia et al. [10] and Youssif et al. [11] which have reported slightly higher detection rates, while the number of operations is higher than Mahfouz [33] which has reported lower detection rates. Basically, our algorithm runs the fastest among those algorithms that provide relatively high detection rates.

In terms of actual processing time, our algorithm processes an image in the STARE database in only $4.1 \mathrm{sec}$ on a $2 \mathrm{MHz}$ Intel Core i5. The method of Youssif et al. [11] as reported in [33] takes $3.5 \mathrm{~min}$ on a $2 \mathrm{MHz}$ Intel Centrino 1.7 CPU. The method of Foracchia et al. [10] as reported in [33] takes $5 \mathrm{~min}$ for vessel extraction on a Sun SPARCstation 20 and 2 min for detection on a $2 \mathrm{MHz}$ Intel Pentium IV. The only faster algorithm reported in the literature is the one by Mahfouz [33] which takes just 0.46 sec on a 2.66 Intel Core2Due, however, as noted above, its reported detection rate is lower than our method with $\mathrm{ONH}$ center error of 14 pixels.

Furthermore, processing of high resolution images in the MUMS-DB (2896 × 1944 pixels) took only around one minute. Our detection algorithm was also found to be scalable in terms of image resolution. We tested it on halfsized retinal images of the three databases. It was found that the ONH detection accuracy still reached $100 \%$ for DRIVE, $96.3 \%$ for STARE, and $95.9 \%$ for MUMS-DB color databases while the processing time was speeded up significantly, that is more than 12 times faster than when using the original resolution.

Note that our method unlike some studies, e.g. [22, 28, 29], does not need to segment retinal vessels for the detection of $\mathrm{ONH}$, rather it relies on the size, intensity, and roundness information. As a result, all the failed retinal images reported in [11] and [10] (i.e., (b) and (d) images in Fig. 13) were correctly detected by our method.

Finally, it is worth stating that our method still has difficulty with a very small or uncommon number of retinal images whose $\mathrm{ONH}$ is darker than the surrounding pixels since it is designed based on the assumption that $\mathrm{ONH}$ is more or less brighter than the surrounding retinal pixels.

\section{CONCLUSION}

In this paper, we have discussed an automated algorithm to detect Optical Nerve Head in a computationally efficient manner. Our algorithm uses a unique combination of Radon Transform and multi-overlapping windows to achieve a robust approach to various conditions such as Diabetic Retinopathy in color fundus images as well as in fluorescein angiography images. Three databases were examined and it was found that our algorithm exhibited high detection rates while achieving higher processing rates than those existing algorithms with comparable detection rates.

\section{ACKNOWLEDGEMENT}

This work was partially supported by the graduate student grant no. 87394 from Mashhad University of Medical Sciences (MUMS). The authors would like to thank the staffs of Khatam-Al-Anbiya Eye Hospital for their assistance with the image acquisition.

\section{REFERENCES}

[1] L. Gagnon, M. Lalonde, M. Beaulieu, and M. C. Boucher, "Procedure to detect anatomical structures in optical fundus images," in Proc. Conf. Med. Imag. 2001: Image Process., San Diego, CA, 2001, pp. 1218-1225.

[2] Y. Tolias and S. Panas, "A fuzzy vessel tracking algorithm for retinal images based on fuzzy clustering," IEEE Trans. Med. Imag., vol. 17, pp. 263-273, Apr. 1998.

[3] C. Sinthanayothin, J. F. Boyce, H. L. Cook, and T. H Williamson, "Automated localisation of the optic disc, fovea, and retinal blood vessels from digital colour fundus images," Br. J. Ophthalmol., vol. 83, no. 8, pp. 902-910, 1999.

[4] A. Osareh, M. Mirmehdi, B. T. Thomas, and R. Markham, "Classification and localisation of diabetic-related eye disease," 7th Eur. Conf.Computer Vision (ECCV), May 2002, vol. 2353, LNCS, pp. 502-516.

[5] A. Osareh, M. Mirmehdi, B. Thomas, and R. Markham, "Automated identification of diabetic retinal exudates in digital colour images," Br. J. Ophthalmol., vol. 87, pp. 1220-1223, 2003.

[6] B. Kochner, D. Schuhmann, M. Michaelis, G. Mann, and K. H Englmeier, "Course tracking and contour extraction of retinal vessels from color fundus photographs: Most efficient use of steerable filters for model-based image analysis," in Proc. SPIE Med. Imag., 1998, pp. 755-761.

[7] A. Aquino, M. E. Gegindez-Arias, and D. Marin, "Detecting the Optic Disc Boundary in Digital Fundus Images Using Morphological, Edge Detection, and Feature Extraction Techniques," IEEE Trans. Med. Imag., vol. 29, no. 11, pp. 1860-1869, 2010.

[8] H. A. Quigley and A. T. Broman, "The number of people with glaucoma worldwide in 2010 and 2020," Br. J. Ophthalmol., vol. 90, pp. 262-267, 2006.

[9] T.Walter and J. C. Klein, "Segmentation of color fundus images of the human retina: Detection of the optic disc and the vascular tree using morphological techniques," in Proc. 2nd Int. Symp. Med. Data Anal., 2001, pp. 282-287.

[10] M. Foracchia, E. Grisan, and A. Ruggeri, "Detection of optic disc in retinal images by means of a geometrical model of vessel structure," IEEE Trans. Med. Imag., vol. 23, no. 10, pp. 1189-1195, Oct. 2004.

[11] A. A. H. A. R. Youssif, A. Z. Ghalwash, and A. R. Ghoneim, "Optic disc detection from normalized digital fundus images by means of a vessels' direction matched filter," IEEE Trans. Med. Imag., vol. 27, pp. 11-18, 2008.

[12] S. Lu and J. H. Lim, "Automatic optic disc detection from retinal images by a line operator," IEEE Trans. Biomed. Eng., vol. 58, pp. 88-94, 2011.

[13] H. Li and O. Chutatape, "Automatic location of optic disc in retinal images," in Proc. IEEE Int. Conf. Image Process. 2001, pp. 837-840. 
[14] H. Li and O. Chutatape, "A model-based approach for automated feature extraction in fundus images," in Proc. 9th IEEE Int. Conf. Comput. Vis. (ICCV'03), 2003, vol. 1, pp. 394-399.

[15] H. Li and O. Chutatape, "Automated feature extraction in color retinal images by a model based approach," IEEE Trans. Biomed. Eng., vol. 51, no. 2, pp. 246-254, Feb. 2004.

[16] A. Osareh, M. Mirmehdi, B. Thomas, and R. Markham, "Comparison of colour spaces for optic disc localisation in retinal images," in Proc.16th Int. Conf. Pattern Recognition, 2002, pp. 743-746.

[17] J. Lowell, A. Hunter, D. Steel, A. Basu, R. Ryder, E. Fletcher, and L. Kennedy, "Optic nerve head segmentation," IEEE Trans. Med. Imag., vol. 23, no. 2, pp. 256-264, Feb. 2004.

[18] J. Xu, O. Chutatape, E. Sung, C. Zheng, and P. C. T. Kuan, "Optic disk feature extraction via modified deformable model technique for glaucoma analysis," Pattern Recognit., vol. 40, no. 7, pp. 2063-2076, 2007.

[19] D. W. K. Wong, J. Liu, J. H. Lim, X. Jia, F. Yin, H. Li, and T. Y. Wong, "Level-set based automatic cup-to-disc ratio determination using retinal fundus images in ARGALI," in Proc. 30th Annu. Int. IEEE EMBS Conf., 2008, pp. 2266-2269.

[20] S. Lu, "Accurate and Efficient Optic Disc Detection and Segmentation by A Circular Transformation," in press, IEEE Trans. Med. Imag., Aug. 2011.

[21] M. Lalonde, M. Beaulieu, and L. Gagnon, "Fast and robust optic disk detection using pyramidal decomposition and Hausdorff-based template matching," IEEE Trans. Med. Imag., vol. 20, no. 11, pp. 1193-1200, Nov. 2001.

[22] F. ter Haar, "Automatic localization of the optic disc in digital colour images of the human retina," M.S. thesis, Utrecht University, Utrecht, The Netherlands, 2005.

[23] K. W. Tobin, E. Chaum, V. P. Govindasamy, and T. P. Karnowski, "Detection of anatomic structures in human retinal imagery," IEEE Trans. Med. Imag., vol. 26, no. 12, pp. 17291739, 2007.

[24] M. Niemeijer, M. D. Abr moff, and B. van Ginneken, "Fast detection of the optic disc and fovea in color fundus photographs," Med. image. anal., vol. 13, no. 6, pp. 859-870, 2009

[25] A. D. Fleming, K. A. Goatman, S. Philip, J. A. Olson, and P. F. Sharp, "Automatic detection of retinal anatomy to assist diabetic retinopathy screening," Phys. Med. Biol., vol. 52, pp. 331-345, 2007

[26] M. Niemeijer, M. D. Abramoff, and B. v. Ginneken, "Segmentation of the optic disc, macula and vascular arch in fundus photographs," IEEE Trans. Med. Imag., vol. 26, no. 1, pp. 116-127, Jan. 2007.

[27] M. Niemeijer, M. D. Abramoff, and B. Van Ginneken, "Automated localization of the optic disc and the fovea," in Conf. Proc. IEEE Eng. Med. Biol. Soc., 2008. pp. 3538-3541.

[28] K. W. Tobin, E. Chaum, V. P. Govindasamy, T. P. Karnowski, and O. Sezer, Reinhardt,M.Joseph, Pluim, and P. W.Josien, Eds., "Characterization of the optic disc in retinal imagery using a probabilistic approach," in Med. Imag. 2006: Image Process., 2006, vol. 6144, pp. 1088-1097.

[29] M. D. Abràmoff and M. Niemeijer, "The automatic detection of the optic disc location in retinal images using optic disc location regression," in Proc. IEEE EMBC 2006, Aug. 2006 pp. 4432-4435.

[30] M. D. Abràmoff, W. L. M. Alward, E. C. Greenlee, L. Shuba C. Y. Kim, J. H. Fingert, and Y. H. Kwon, "Automated segmentation of the optic disc from stereo color photographs using physiologically plausible features," Invest. Ophthalmol. Vis. Sci., vol. 48, no. 4, pp. 1665-1673, 2007.

[31] H. K. Hsiao, C. C. Liu, C. Y. Yu, S. W. Kuo, S. S. Yu., "A novel optic disc detection scheme on retinal images," Expert Systems with Applications, vol. 39, pp. 10600-10606, 2012.

[32] H. Yu, E. S. Barriga, C. Agurto, S. Echegaray, M. S. Pattichis W. Bauman, P. Soliz, "Fast Localization and Segmentation of Optic Disk in Retinal Images Using Directional Matched Filtering and Level Sets," IEEE Transactions on Information Technology in Biomedicine, vol. 16, no. 4, pp. 644-657, 2012.

[33] A. E. Mahfouz and A. S. Fahmy, "Fast localization of the optic disc using projection of image features," IEEE Trans. Imag. Process., vol. 19, pp. 3285-3289, 2010.

[34] J. Lowell, A. Hunter, D. Steel, A. Basu, R. Ryder, and R. L. Kennedy, "Measurement of retinal vessel widths from fundus images based on 2-D modeling," IEEE Trans. Med. Imag., vol. 23, no. 10, pp. 1196 1204, Oct. 2004

[35] J. Staal, M. D. Abramoff, M. Niemeijer, M. A. Viergever, and B. V. Ginneken, "Ridge-based vessel segmentation in colo images of the retina," IEEE Trans. Med. Imag., vol. 23, no. 4, pp. 501-509, Apr. 2004

[36] A. Hoover and M. Goldbaum, "Locating the optic nerve in a retinal image using the fuzzy convergence of the blood vessels," IEEE Trans. Med. Imag., vol. 22, no. 8, pp. 951-958, Aug. 2003. 